\title{
Anticancer Potential of Cephalotaxine and Derivatives as an Alternative for Invasive Therapies
}

\author{
Evangelina Kalathoti ${ }^{1, \#}$, Heer Nanda ${ }^{2, \#}$, Riya Parekh3,\#, Aditi Shankar ${ }^{1, \#,}$

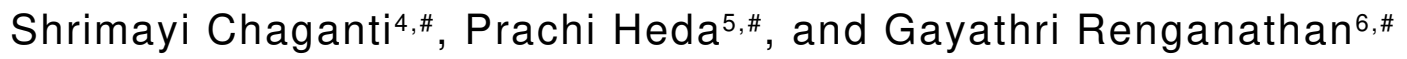 \\ ${ }^{1}$ Mission San Jose High School, Fremont, CA, USA \\ ${ }^{2}$ Amador Valley High School, Pleasanton, CA, USA \\ ${ }^{3}$ Irvington High School, Fremont, CA, USA \\ ${ }^{4}$ Monta Vista High School, Cupertino, CA, USA \\ ${ }^{5}$ Dougherty Valley High School, San Ramon, CA, USA \\ ${ }^{6}$ Anna University, Chennai, TN, India \\ \#Aspiring Scholars Directed Research Program (ASDRP), Fremont, CA, USA \\ DOI: https://doi.org/10.47611/jsrhs.v10i2.1483
}

\section{$\underline{\text { ABSTRACT }}$}

Cancer is identified by the abnormal accumulation of rapidly dividing cells, serving as the collective term for a multitude of similar conditions categorized through cell growth and proliferation. The disease continues to lead death tolls worldwide and extends to communities across the board in terms of socioeconomic status.(^1) From 1991 to 2017, experts recorded a slight drop in cancer death rates, which can be accredited to an influx of scientific advancements within the past decade that allowed for the implementation of targeted therapy. $\left({ }^{\wedge} 1\right)$ In preclinical research, this therapy implies the conjugation of independent drugs retaining anti-carcinogenic properties. The current state of cancer pathology is observed to be non-specific and is, oftentimes, taxing on the patient, proving the need for localized treatment and more efficient drugs. This article analyzes the effectiveness of one compound under the harringtonine alkaloid family, cephalotaxine (CET).(^27) In this work, our team examines the biochemical profile of CET and congener homoharringtonine to hypothesize cephalotaxine's efficacy as an alternative to invasive cancer therapy.

\section{Introduction}

Cephalotaxine, along with an array of other compounds, is a secondary metabolite of Cephalotaxus Harringtonia, a coniferous plant belonging to the family Taxaceae. ${ }^{3}$ Secondary metabolites are nonessential supplements in the body and fall into three categories: phenolics, which contain hydroxylated aromatic rings, terpenoids, which are derivatives of polymeric isoprenes and naturally synthesized through the mevalonic acid pathway, and alkaloids, a class of nonprotein compounds containing nitrogen. ${ }^{2}$ These metabolites serve as promising anti-cancer agents, and plant metabolites, among which is cephalotaxine, have the ideal composition needed for clinically tested compounds. Cephalotaxine has a chemical formula of $\mathrm{C} 18 \mathrm{H} 21 \mathrm{NO} 4$. As an optically active compound, cephalotaxine would typically be derived from the organic acid proline as conducted in a Seebach protocol, amounting to a molecular weight of 315.4 $\mathrm{g} / \mathrm{mol}$ once formed. ${ }^{30}$ As a polycyclic alkaloid, CET has a unique structural orientation; it contains a 1azaspiro[4.4]nonane ring system, making the compound a prime candidate for total synthesis. ${ }^{9}$ The heterocyclic $\mathrm{ABCDE}-$ ring skeleton of cephalotaxine, a spirane compound, continues to be an area of focus in research, as it may provide insight into more effective synthesis methods. ${ }^{9}$ There are no definitive origins of the formation of cephalotaxine, but there are a number of theories. Some scientists suggest that cephalotaxine materializes when two molecules 
of tyrosine condense to produce B-phenethylamine molecules, which are then cyclized to yield a tetrahydro benzazepine. ${ }^{5}$ Following that, oxidative fission of the aromatic rings affords the keto-acid, ultimately yielding cephalotaxine. ${ }^{5}$

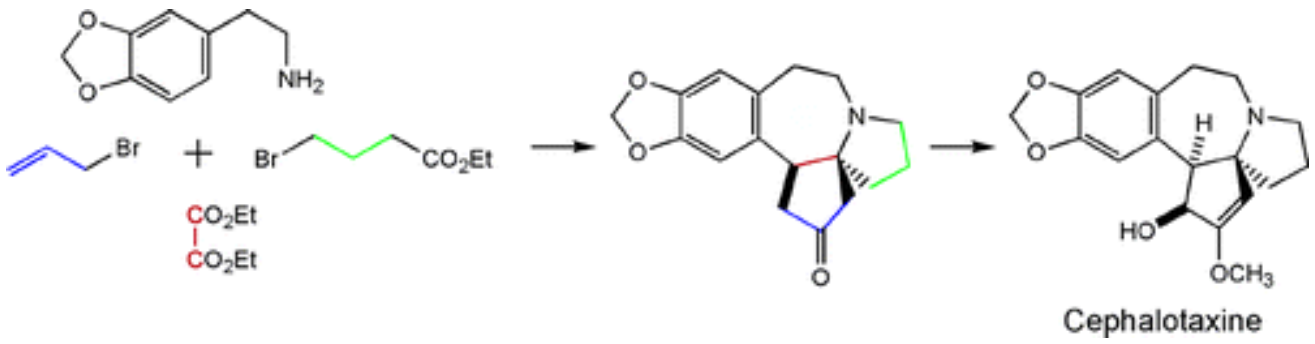

Fig 1 Formation of cephalotaxine's pentacyclic ring through synthetic transannular reductive skeletal rearrangement as a novel approach to total synthesis. ${ }^{9}$

Besides cancer therapy, cephalotaxine has shown promise as an anti-Flavivirus medication. The Zika Virus (ZIKV) is a mosquito-borne flavivirus that was declared to be a Public Health Emergency of International Concern by the WHO. ${ }^{4}$ Currently, no approved antiviral drugs are available for ZIKV, so the need to develop antiviral agents is urgent. It has been found that CET possesses anti-ZIKV activity by exhibiting virucidal activity and preventing ZIKV from replicating. ${ }^{4}$ When CET was administered to ZIKV infected Vero cells, Western blotting analysis revealed that ZIKV protein expression reduced significantly, and FFU assays revealed that CET inhibits ZIKV in a dose-dependent manner. ${ }^{4}$ After additional assays were performed, the results gave evidence that CET suppresses viral RNA replication and affects virus stability. ${ }^{4}$ These findings suggest that CET has the potential to be developed as a therapeutic agent against ZIKV. ${ }^{4}$

Cephalotaxine manifests its properties into ester derivatives, such as homoharringtonine (HHT/Omacetaxine mepesuccinate). ${ }^{3}$ CET is a functional parent of Omacetaxine, a protein translation inhibitor that has been used to treat Chronic Myeloid Leukemia, since it is resistant to tyrosine kinase receptor antagonists. ${ }^{6}$ Normal protein translation occurs in three steps on ribosomes. There are three sites in the ribosome: the A, P, and E sites. First, when the tRNA (Transfer-RNA) linked with the amino acid comes in for the translation process, it binds to the matching mRNA at the acceptor (A site). ${ }^{7}$ Then, it transfers the amino acid to the incoming amino acid and moves to the peptidyl-tRNA ( $\mathrm{P}$ site), allowing the following tRNA to bring in the next amino acid and continue protein translation/production. ${ }^{7}$ The resulting deacylated tRNA is moved to the E site and is separated from the ribosome. ${ }^{7}$ Translation (protein synthesis) is inhibited by the use of omacetaxine, a competitive inhibitor that competes with tRNA to bind to the A site. ${ }^{7}$ Once omacetaxine has covalently bonded to the acceptor site, no other tRNA's are able to bind, preventing further production of proteins, especially those with small half-lives. ${ }^{7}$ The cells that are reliant on the proteins produced by translation undergo apoptosis, preventing the further growth of chronic myeloid leukemia cells, promoting tumor regression. $^{7}$

Homoharringtonine (HTT), a derivative of cephalotaxine, is a plant alkaloid derived from Cephalotoxus fortunei. It has been shown to exhibit strong antiviral activity against herpes viruses, coronaviruses, rhabdoviruses, and more. In this particular study, the objective was to evaluate the in-vitro antiviral effect of several compounds (including HTT) that were previously reported to inhibit coronavirus replication and compounds that are currently in clinical trials dealing with SARS-CoV-2 patients. ${ }^{8}$ Vero E6 cells were infected with the SARS-CoV-2 virus and then treated with homoharringtonine. It was observed that HTT inhibits SARS-CoV-2 with an EC50 value of $2.10 \mathrm{uM}$, reflecting the compound's potential in conditions beyond solely malignancies. ${ }^{8}$ 


\section{Epidemiology of Cancer}

In studying the epidemiology of cancer, three types of epidemiological research applies: descriptive, analytic, and clinical epidemiology. Descriptive epidemiology focuses on the trend pattern and frequency of cancer or any disease in a particular population. The American Cancer Society estimates by the end of 2020, there will be an estimated 1.8 million new cancer cases and 606,520 cancer deaths in the United States. Prostate cancer is the most common among males, followed by lung, and colorectal cancers. Among females, breast, lung, and colorectal cancers are the most common. Among males, lung cancer is the leading cause of death, followed by prostate and colorectal cancers. For females, lung, breast, and colorectal cancers are the leading causes of death. The current average annual incidence rate of cancer from 2012 to 2016 is $16 \%$ higher in men than in women. ${ }^{13}$ During the most recent 10 years of data collected, the overall cancer incidence rate declined rapidly until 2014 and stabilized in 2016, showing slow declines for colorectal cancer and the stabilizing of prostate cancer. On the other hand, the cancer incidence rate for women has remained steady over the last decade. Furthermore, the American Cancer Society estimates that cancer incidence rates are higher in males than in females in every racial or ethnic population, with the highest incidence rates for cancer in Non-Hispanic Blacks among males and in Non-Hispanic Whites in females, while mortality rates rank highest for Non-Hispanic Black males and females.

The goal of analytic epidemiology is to identify the factors that are responsible for the development of a disease, and in this case, cancer. Risk factors connected to the development of cancer include alcohol, tobacco, diet, and viruses or infections. In 2012, the IARC Working Group classified alcoholic beverages as carcinogenic to humans and concluded that many different types of cancers are related to the consumption of alcohol. Due to limited evidence, a concurrent meta-analysis, including more than 500 studies from across the globe and nearly half a million cancer cases, was conducted to observe associations between alcohol consumption and 23 different cancer sites. Data showed that dose-response relationships showed the highest risk among heavy drinkers as compared with nondrinkers and occasional drinkers. In addition, tobacco and electronic cigarettes were shown to have a relation in the development of cancer and cause premature disease and death in the U.S. ${ }^{14}$ A report from 2014, documenting 50 years of evidence after the initial report found that numerous cancers including cancers of the head and neck, bronchus and lung, stomach, liver, pancreas, kidney and ureter, cervix, bladder, colon, and rectum, were linked to smoking.$^{15}$ Furthermore, it showed that not only were tobacco users at risk of the development of cancers but were also at an increased risk for treatment complications. A comprehensive report on Food, Nutrition and Physical Activity and the Prevention of Cancer published by the World Cancer Research Fund (WCRF) and American Institute for Cancer Research (AICR) classified certain foods on a scale, many of which that had the ranking convincing or probable increased risk, showing that diet can also be associated with cancer. High-risk foods including red meat, processed meat, and alcoholic drinks were connected to various types of cancers. On the other hand, the scale also classified many foods as convincing or probable decreased risk too. Disease and infections, usually regarded as distinct disciplines, have, in recent years, shown more links to each other. Currently, at least seven viruses such as Epstein-Barr virus, hepatitis B virus (HBV), and HPV have been associated with cancer risk.

Clinical epidemiology plays an important role in cancer screening modalities and prevention strategies by lowering risk through lifestyle and behavior. Cancer screening is a method that involves testing to detect cancer at an early stage in asymptomatic patients. Cancer screening is very beneficial, as an early detection of cancer will improve patient survival and treatment. Chemoprevention, a novice method compared to cancer screening, is the reversal of carcinogenesis in the premalignant phase. ${ }^{16}$ In the process of chemoprevention, doctors use natural or lab made chemoprevention drugs to lower the risk of a person developing cancer. In most cases, chemoprevention drugs are not used to treat cancer. Some chemoprevention drugs include tamoxifen (Soltamox) and raloxifene (Evista). Chemotherapy is a method used to treat and destroy cancer after it appears in the body. Some chemotherapy drugs include asparaginase, procarbazine, and many more. ${ }^{17}$ 


\section{Biochemistry of Cancer}

Cancer originates when one cancerous cell multiplies through the cell cycle and goes undetected by correcting enzymes or by the immune system. ${ }^{18}$ The chance of a mutation occurring is $10^{6}$ which is why cancer is generally concentrated in the older populations. ${ }^{18}$ One factor that contributes to cancer includes carcinogens such as X-rays, UV radiation, and tobacco. Effective carcinogens found in cow milk such as methylcholanthrene and aflatoxins, can lead to hepatoma. ${ }^{18}$ These carcinogens can alter DNA through covalent binding; the N2, 3, and 7 atoms of guanine are more susceptible to have carcinogen groups bound to them. ${ }^{18}$ The resulting oligomer is called a dimer, which causes DNA breakages, leading to mutations. ${ }^{18}$ When mutated, proto-oncogenes such as TGF or p53 can turn into oncogenes. ${ }^{18}$ From oncogenes, we can obtain growth factors, receptors, or phosphorylates which can activate cascades. ${ }^{18}$ The human body takes protective measures against various cancers. For example, oncosuppressors such as the p53 genes act against conditions including retinoblastoma, wilms tumor, familial adenomatous polyposis, and familial breast cancer. ${ }^{18}$ Additionally, there are a plethora of natural antimutagens, ranging from vitamins $\mathrm{A}, \mathrm{C}, \mathrm{E}$ to curcumin that act as antioxidants or inhibit the production of new tumor cells. Transformation, the process in which a virus integrates its DNA into the host's to multiply, can prompt malignant cell transformation, leading to cancerous growth. ${ }^{18}$ Oncofetal proteins induce cancer and are suppressed during the differentiation process, but in the case of a malignant transformation, they can be reactivated and become harmful proteins. ${ }^{18}$ These proteins are also known as tumor index substances, so they can be used to test for cancer post-cancer therapy. ${ }^{18}$ Cytotoxic drugs are used as a preventative measure for cancer, and affects all cells in the diving phase, causing collateral damage to healthy cells. ${ }^{18}$

Ras and p53 are common genes related to cancer. Ras is associated with GTP/GDP cycle and regulates cell signaling pathways. ${ }^{19}$ P53 is a tumor suppressor gene that helps detect errors in DNA sequences. ${ }^{19}$ After p53 is activated, it can lead to apoptosis and other mechanisms used during the termination of cancerous cells. ${ }^{19}$ In the cell cycle, the Ras gene plays a big role since it promotes the development of the cell and stops the tumorous cells from growing. ${ }^{19}$ Ras releases either CDK inhibitors or proteins that affect cell growth during the G1/G2 phases. ${ }^{19}$ Other possible treatment regulations of cancer can be implemented by release of protein inhibitors during the cell cycle. One example includes PKA, which is released and used for cell differentiation. ${ }^{19}$ Cancer, however, causes PKAI to be overexpressed, so the downregulation of that can be used as a possible treatment. ${ }^{19}$

\section{Cephalotaxine Profile}

\section{Pathways of Cephalotaxine}

Research on cephalotaxine's effect on signal pathways is limited. However, there is significant research on the compound's derivative, homoharringtonine (HTT). In China, HTT was effective in treating patients with acute myeloid leukemia (AML) but there is limited information about the mechanism of its action. ${ }^{20}$ The objective of this study was to investigate the effect of HTT on JAK2-STAT in AML cells as the abnormal activation of JAK2 associated pathway is important to AML. RT-PCR was used to measure the expression of JAK 2, STAT5, and affect gene Bcl-xL. Signal proteins such as p-JAK2, p-STAT5, p-AKT, p-ERK that were activated by the abnormal activated JAK2 were tested by western blotting. Homoharringtonine inhibited the viability of primary AML cells and AML cell lines. The double staining of AnnexinV-P confirmed early apoptosis in treatment that were dependent on doses. When AML cells were affected by HTT for 6 hours, the expressions of p-JAK2, p-STAT5, p-AKT, p-ERK were down-regulated, while the protein levels of JAK2, STAT5, and AKT were stable. HHT possibly acts as a broad-spectrum PTK inhibitor and inhibits the phosphorylation of the signal proteins. This blocks the survival and proliferative signal pathways of malignant cells.

One of the most common types of gastrointestinal tumors is the colorectal cancer (CRC) which is characterized by a poor survival rate. ${ }^{31}$ For the treatment of acute myeloid leukemia and chronic myeloid leukemia, HTT is 
approved, but for CRC, the effects of single-use of HTT are not known. It seems as though EphB4 is abnormally overexpressed in patients diagnosed with CRC. This led to the objective of the study:study the inhibitory effect of HTT in CRC and its mechanism. LoVo cell growth was suppressed by HTT in vitro and in vivo. HTT also induced apoptosis and cell cycle arrest at the $\mathrm{S}$ phase. Using western blotting revealed that HTT suppressed EphB4, and this suppression was enlarged by the co-administration of HTT and NVP-BHG712. This indicates that HTT targets EphB4 to stop LoVo cell growth. HHT inhibited EphB4 downstream pathways such as PI3K/AKT and MAPK/EKR1/2 which resulted in the regulation of cell-cycle related molecules and the molecules in the Bcl-2 mitochondrial apoptosis pathway. For this reason, HTT seems to be a promising EphB4 inhibitor and HTT has great potential for CRC treatment.

\section{Role of Drug (effectiveness of drug based on past research)}

Cephalotaxus alkaloids have been researched since the 1960's for their potency against the Zika virus, Hepatitis B, and multiple types of cancers through its synthetic, semisynthetic, and natural compositions. ${ }^{12}$ Two of its main derivatives, cephalotaxine (CET) and homoharringtonine (HHT), have been reported as promising antitumor agents, and in China, HHT has been widely used for the treatment of chronic myeloid leukemia (CML), acute myeloid leukemia (AML), and myelodysplastic syndrome (MDS). The semisynthetic form of HHT, omacetaxine, has also displayed high bioavailability through the subcutaneous route, and was approved by the U.S FDA for treatment of CML. ${ }^{6}$

Specifically, HHT inhibits protein synthesis at the translation site of ribosomes by preventing the elongation phase via an interaction with ribosomal A-site. It triggers the rapid loss of short-lived proteins that regulate cell proliferation/survival of several hematological malignant cell lines, resulting in HHT-induced apoptosis. To test its efficacy against various refractory cancers, HHT has undergone clinical trials in the United States. During the phase I clinical trials of homoharringtonine, 43 patients with malignancies were administered with a daily iv treatment for 5 days at intervals of 3 to 4 weeks. $^{21}$ The initial dose of homoharringtonine was $0.2 \mathrm{mg} / \mathrm{m} 2 /$ day which gradually increased to a maximum of $8 \mathrm{mg} / \mathrm{m} 2 /$ day. Dosage levels of $3-4.5 \mathrm{mg} / \mathrm{m} 2 /$ day induced mild hypotension in patients which was generally treated by iv fluid supplements. As dosage amounts escalated, hypotension became increasingly severe, resulting in cardiovascular collapse of four out of 16 patients who received HHT quantities of 5-6 mg/m2/day. Patients treated with doses equivalent to or greater than $3 \mathrm{mg} / \mathrm{m} 2$ exhibited moderately-acute degrees of myelosuppression. The intensity and prevalence of myelosuppression correlated with the extent of prior treatment received, and was marginal in patients that received little to no prior treatment. Approximately two-thirds of the patients were observed to have the mild gastrointestinal toxic effects of nausea, vomiting, and diarrhea, along with drug-related side effects of fever and alopecia. Three patients with solid tumors showed minor degrees of response and three out of five patients with acute leukemia manifested moderate antileukemic activity. From obtaining these results, phase II studies of HHT were precisely curated to avoid high symptomatic expression of hypotension/myelosuppression.

\section{Pharmacokinetics \& Pharmacodynamics of Cephalotaxine and Homoharringtonine}

The lack of accessible literature due to language barriers and a shortage of research speculating the pharmacokinetics of CET and HHT is responsible for a deficiency in understanding. ${ }^{25}$ However, it is known that both cephalotaxine and homoharringtonine play an important anticancer role in the body. ${ }^{26}$ They are more active in cell lines without the p53 mutation as p53 affects the response of tumor cells to cephalotaxine and homoharringtonine. ${ }^{26}$ Homoharringtonine is known for its high growth inhibitory activity towards human leukemic cells. ${ }^{26}$ More specifically, it inhibits protein production, allowing activity against the production of acute and chronic myeloid leukemias. ${ }^{26}$ On the $60-\mathrm{S}$ ribosome, HHT prevents the substrate from binding to the receptor site, blocks aminoacyl-tRNA binding, and peptide bond formation, suppressing the elongation phase of translation. ${ }^{26} \mathrm{HHT}$ also prevents lipid-linked oligosaccharide formation and glycosylation of glycoproteins. ${ }^{26}$ Furthermore, homoharringtonine suppresses the incorporation of [3H] thymidine into DNA. ${ }^{26}$ Although the inhibition of DNA synthesis is a secondary process when compared to the prevention of 
protein biosynthesis, both lead to the stopping of the cell cycle in the G and G2 phases and prompt apoptosis. ${ }^{26}$ Further, in a study done administering cephalotaxine and homoharringtonine on human jurkat cells, in comparison to CET, HHT was four times greater in magnitude than cephalotaxine, meaning that it was more active and had a greater effect on the cells in lesser dosages than what was needed for cephalotaxine. ${ }^{26}$

HHT and CET are also associated with an array of genes: DNA damage and repair genes, drug resistant genes, cytokines related genes, apoptosis-regulation genes, and proliferation-associated genes. ${ }^{26}$ In a specific study, it was found that several apoptosis-regulating genes are connected to the cellular responses of CET and HHT, showing us that HHT can induce apoptosis and activate caspases. ${ }^{26}$ When mutated, p53 provides cellular resistance to CET and HHT. ${ }^{26}$ Since drug resistance can be multifactorial, some CET-unresponsive cell lines did not have any p53 mutations, confirming this concept. ${ }^{26}$ Other oncogenes and tumor suppressor genes such as EGFR were correlated with the activity of cephalotaxine and homoharringtonine as well. ${ }^{26}$

Finally, HHT has shown high activity against colon 38 tumor and P388 leukemia, mild activity against CD8F mammary carcinoma and L1210 leukemia, and little to no activity against B16 melanocarcinoma. ${ }^{22}$ To test the viability of HHT in mice with injected P388 leukemia cells, HHT was administered at a dose of $2 \mathrm{mg} / \mathrm{kg}$ daily for 9 days, resulting in a $140 \%$ increase in lifespan of the mice. ${ }^{22}$ When HHT was used in sygnersim with other compounds, it was highly active against leukemic cells from patients with CML-BP. ${ }^{22}$

\section{Discussion}

\section{Structural activity}

Cephalotaxine (I) is a benzazepine alkaloid isolated from Cephalotaxus harringtonia. It is a benzazepine alkaloid fundamental parent, an organic heteropentacyclic compound, an enol ether, and a cyclic acetal. ${ }^{10}$ This group of alkaloids is of interest due to its unusual structure, having an azaspiro system fused to an azepine ring, and its potent anticancer activity. The fractionation of a seed extract obtained from C. harringtonia (Forbes) K. Koch var. harringtonia cv. Fastigiata (entire plants) revealed four alkaloids with significant antitumor activity: harringtonine (II), isoharringtonine (III), homoharringtonine (IV), deoxyharringtonine (V), and acetylcephdotaxine (VII). ${ }^{23}$ When the nuclear magnetic resonance (NMR) spectra was observed, it yielded evidence that the compounds, II-V, were esters of cephalotaxine. When all $\mathrm{R}$ groups were removed from the esters and compared, the NMR spectra became almost identical to each other.

All of the cephalotaxus alkaloids were tested against G1210 or P388 leukemia, and the relative survival times of treated and control animals made it evident that alkaloids 11-IV have a greater activity against G1210, while cephalotaxine (I) and acetylcephdotaxine (VII) were inactive. ${ }^{24}$ Even greater activity was shown from alkaloids II-V against P388 leukemia taking into account the wide range of dosage levels. As mentioned above, these compounds differ only in the ester (R) group, and therefore slight modification of this affiliation can significantly be shown to have affects antitumor activity. Additional synthesis of other cephalotaxine esters with different $\mathrm{R}$ groups can also lead to compounds having even more desirable antitumor properties than the esters mentioned above.

Cephalotaxine (I), when crystallized by slow evaporation, yielded a mass spectrum of ions at at m/e 315 (M+, 1OOx), 300 (54), 298 (57), 284 (67), 272 (17), 254 (15), 214 (19), 166 (36), 150 (23), 137 (26), and 115 (16).23 In a dimethyl sulfoxide solution, cephalotaxine was shown to exhibit a one-proton doublet, at $\delta 4.78$, which is coupled to H-3 (q. $\delta$ 4.51). After exchange in deuterium oxide, only the H-3 signal is apparent (d, 64.51 ). An authentic sample of cephalotaxine (2), recrystallized from ether,gavem.p. 135-136", [a]D -2110(cO.04inethanol).The NMR, IR, and UV spectra of this material, as well as the corresponding spectra of all cephalotaxine samples isolated in the study, were indistinguishable. 


\section{Conclusion}

Our team recommends conducting further studies on cephalotaxine and its derivatives to ultimately determine its effect on malignancies. Testing may include, but is not limited to, in vitro administration of CET and HHT against human mammalian tumor cell lines to assess the rate of tumor regression as induced by the compound. Studies examining the potential of HHT against colorectal cancer can provide insight beyond hematological cancers. Synergistic trials are another avenue for future testing as CET and HHT have shown prospective results in synergism with phytochemicals, such as sulforaphane, and other anticancer agents. Combination drugs are often administered in the form of liposomes, serving as an effective method. Exploring synthetic strategies would increase treatment potential and aid applicability, improving final therapeutic outcome. From this research, CET, along with its derivatives, displays potency against various malignancies and viruses, while holding promising potential in treating other subtypes of leukemia, CRC, HIV, and many more diseases.

\section{Acknowledgments}

The authors would like to thank Aspiring Scholars Directed Research Program (ASDRP) and Ms. Gayathri Renganathan for their help on this project.

\section{References}

1. Siegel, R.L., Miller, K.D. and Jemal, A. (2020), Cancer statistics, 2020. CA A Cancer J Clin, 70: 7-30. https://doi.org/10.3322/caac.21590

2. Seca, A. M. L. (2018, January 16). Plant Secondary Metabolites as Anticancer Agents: Successes in Clinical Trials and Therapeutic Application. PubMed. https://pubmed.ncbi.nlm.nih.gov/29337925/

3. Cephalotaxine - an overview | ScienceDirect Topics. (1998). ScienceDirect. https://www.sciencedirect.com/topics/neuroscience/cephalotaxine

4. Lai ZZ, Ho YJ, Lu JW. Cephalotaxine inhibits Zika infection by impeding viral replication and stability. Biochemical and Biophysical Research Communications. 2020 Feb;522(4):1052-1058. DOI: 10.1016/j.bbrc.2019.12.012.

5. Biosynthesis. (n.d.). Google Books. https://books.google.nl/books?id=fnIoDwAAQBAJ\&pg=PA142\&lpg=PA142\&dq=cephalotaxine+biosynthesis\&source=bl\&ots=2H1JoX2i2m\&sig $=$ ACfU3U3IQFJbYk8kC06XNwufgCR7jAD2XA\&hl=en\&sa=X\&redir_esc $=y \# v=$ onepage $\& q=$ cephalotaxi ne $\% 20$ biosynthesis $\& \mathrm{f}=$ false

6. Gandhi, V. G., Plunkett, W. P., \& Cortes, J. C. (2014, February 5). Omacetaxine: a protein translation inhibitor for treatment of chronic myelogenous leukemia. NCBI. https://www.ncbi.nlm.nih.gov/pmc/articles/PMC4048124/

7. Winer, E. S., \& DeAngelo, D. J. (2018, March 15). A Review of Omacetaxine: A Chronic Myeloid Leukemia Treatment Resurrected. Oncology and Therapy. https://ink.springer.com/article/10.1007/s40487-0180058-6?error=cookies_not_supported\&code=a33353a8-94e1-4b91-9e74-9878373c091e

8. Choy, K. T., Wong, A. Y., Kaewpreedee, P., Sia, S. F., Chen, D., Hui, K., Chu, D., Chan, M., Cheung, P. P., Huang, X., Peiris, M., \& Yen, H. L. (2020). Remdesivir, lopinavir, emetine, and homoharringtonine 
inhibit SARS-CoV-2 replication in vitro. Antiviral research, 178, 104786. https://doi.org/10.1016/j.antiviral.2020.104786

9. Wang, Y. W., \& Li, W. L. (2003, June 16). A Novel and Efficient Total Synthesis of Cephalotaxine. http://www.paper.edu.cn/scholar/showpdf/MUT2ANyIMTz0MxOh

10. National Center for Biotechnology Information (2020). PubChem Compound Summary for CID 65305, Cephalotaxine. Retrieved December 30, 2020 from

11. Efferth, T., Sauerbrey, A., Halatsch, M., Ross, D. D., \& Gebhart, E. (2003, January 1). Molecular modes of action of cephalotaxine and homoharringtonine from the coniferous tree Cephalotaxus hainanensis in human tumor cell lines. Naunyn-Schmiedeberg's Archives of Pharmacology. https://link.springer.com/article/10.1007/s00210-002-0632-0?error=cookies_not_supported\&code=cc30da22-977e-4404-9805$85793 \mathrm{~d} 88218 \mathrm{c}$

12. Pérard-Viret J, Quteishat L, Alsalim R, Royer J, Dumas F. Cephalotaxus Alkaloids. The Alkaloids. Chemistry and Biology. 2017;78:205-352. DOI: 10.1016/bs.alkal.2017.07.001.

13. American Cancer Society. Cancer Facts \& Figures 2020. Atlanta: American Cancer Society; 2020.https://www.cancer.org/research/cancer-facts-statistics/all-cancer-facts-figures/cancer-facts-figures2020.html

14. Arem, H., \& Loftfield, E. (2017). Cancer Epidemiology: A Survey of Modifiable Risk Factors for Prevention and Survivorship. American journal of lifestyle medicine, 12(3), 200-210. https://doi.org/10.1177/1559827617700600

15. U.S. Department of Health and Human Services. The Health Consequences of Smoking-50 Years of Progress: A Report of the Surgeon General. Atlanta, GA: U.S. Department of Health and Human Services, Centers for Disease Control and Prevention, National Center for Chronic Disease Prevention and Health Promotion, Office on Smoking and Health, 2014.

16. Nazario, A., Macheledt, J., \& Vogel, V. (1995, March 31). Epidemiology of Cancer and Prevention Strategies. Retrieved December 30, 2020, from https://www.cancernetwork.com/view/epidemiology-cancer-andprevention-strategies

17. How Chemotherapy Drugs Work. (2019). Retrieved December 30, 2020, from https://www.cancer.org/treatment/treatments-and-side-effects/treatment-types/chemotherapy/how-chemotherapy-drugswork.html

18. Vasudevan, Damodaran \& S, Sreekumari \& Vaidyanathan, Kannan. (2017). Chapter-48 Biochemistry of Cancer. 10.5005/jp/books/13014_49.

19. Isoldi, Mauro \& Visconti, Maria \& Castrucci, Ana. (2005). Anti-Cancer Drugs: Molecular Mechanisms of Action. Mini reviews in medicinal chemistry. 5. 685-95. 10.2174/1389557054368781.

20. Tong, Hongyan \& Ren, Yanling \& Zhang, Fengjuan \& Jin, Jie. (2008). Homoharringtonine affects the JAK2-STAT5 signal pathway through alteration of protein tyrosine kinase phosphorylation in acute myeloid leukemia cells. European journal of haematology. 81. 259-66. 10.1111/j.1600-0609.2008.01116.x.

21. Legha SS, Keating M, Picket S, et al. Phase I clinical investigation of homoharringtonine. Cancer Treatment Reports. 1984 Sep;68(9):1085-1091. 
22. Quintás-Cardama, A., Kantarjian, H. and Cortes, J. (2009), Homoharringtonine, omacetaxine mepesuccinate, and chronic myeloid leukemia circa 2009. Cancer, 115: 5382-5393. https://doi.org/10.1002/cncr.24601

23. Powell, R., Weisleder, D., \& Smith, C. (1972). Antitumor Alkaloids from Cephalotaxus harringtonia: Structure and Activity. Journal of Pharmaceutical Sciences, 61(8), 1227-1230.

doi:10.1002/jps.2600610812

24. Chang, Y., Meng, F., Wang, R., Wang, C., Lu, X., \& Zhang, Q. (2017). Chemistry, Bioactivity, and the Structure-Activity Relationship of Cephalotaxine-Type Alkaloids From Cephalotaxus sp. Studies in Natural Products Chemistry, 53, 339-373. doi:10.1016/b978-0-444-63930-1.00010-

25. Lu, K., Savaraj, N., Feun, L. G., Zhengang, G., Umsawasdi, T., \& Loo, T. L. (1988, April 1). Pharmacokinetics of homoharringtonine in dogs. Cancer Chemotherapy and Pharmacology. https://link.springer.com/article/10.1007/BF00257360?error=cookies_not_supported\&code=2c67adc04fc9-4fdc-a16d-b25d173db6a8

26. Efferth, T., Sauerbrey, A., Halatsch, M., Ross, D. D., \& Gebhart, E. (2003b, January 1). Molecular modes of action of cephalotaxine and homoharringtonine from the coniferous tree Cephalotaxus hainanensis in human tumor cell lines. Naunyn-Schmiedeberg's Archives of Pharmacology. https://link.springer.com/article/10.1007/s00210-002-0632-0?error=cookies_not_supported\&code=a7ed3ec5-c6dc-4080-9d8bd8331c0ab999

27. J, P., L, Q., R, A., J, R., \& F, D. (2017, August 16). Cephalotaxus Alkaloids. Abstract - Europe PMC. http://europepmc.org/article/MED/28838429\#s0380

28. P. (n.d.). Cephalotaxine. PubChem. https://pubchem.ncbi.nlm.nih.gov/compound/Cephalotaxine\#section=Toxicity

29. Simon, S. (2020, January 8). Facts \& Figures 2020 Reports Largest One-year Drop in Cancer Mortality. American Cancer Society. https://www.cancer.org/latest-news/facts-and-figures-2020.html

30. Total Synthesis of (-)-Cephalotaxine. (n.d.). Retrieved January 14, 2021, from https://pubs.acs.org/doi/10.1021/jo00106a023

31. Shi, X., Zhu, M., Gong, Z., Yang, T., Yu, R., Wang, J., \& Zhang, Y. (2019, November 11). Homoharringtonine suppresses LoVo cell growth by inhibiting EphB4 and the PI3K/AKT and MAPK/EKR1/2 signaling pathways. Retrieved January 14, 2021, from https://www.sciencedirect.com/science/article/abs/pii/S0278691519307501\# 